\title{
Fortalecimiento de hábitos de estudio a través de las tecnologías de la información y la comunicación ${ }^{1}$
}

\section{Strengthening study habits through information and communication technologies}

DOI: http://dx.doi.org/10.17981/cultedusoc.9.3.2018.20

Artículo de investigación. Fecha de recepción: 15/06/2018. Fecha de aceptación: 27/11/2018

Pablo Salcedo Paternóstro; Oscar Vargas Montalva; Asdrubal Urrea Pacibes;

Hernando Pérez V.; Reinaldo Gonzales Pérez; Zulay Martinez Mosquera;

Nadin Caballero Ternera; Ibis Castañeda Parra; José Lobato Lobo;

Heiman Charris Cantillo; Maribel Cantillo V.; Elsy Cantillo Escorcia;

Edison Lobato Lobo; Olga Álvarez Lobo; Amelia Vargas Morales;

Nélida De Jesús Africano; Denis Lobato Barrios; Yolanda Villalobos;

Leribeth Polo González; Hernando Pérez Villamil; Rafael Gómez Manga;

Fareth Altamar; Jorge Álvarez Cantillo; Ausberto González y Carlos Carbono Lobato²

Institución Educativa Departamental Rural de Media Luna,

sedes Erm Varones De San José, colegio rural de media luna y Erm Niñas Del Socorro (Colombia)

Para citar este artículo:

Salcedo, P., Vargas, O., Urrea, A., Pérez, H., Gonzales, R., Martinez, Z., Caballero, N., Castañeda, I., Lobato, L., Charris, H., Cantillo, M., Cantillo, E., Lobato, E., Álvarez, O., Vargas, A., De Jesús, N., Lobato, D., Villalobos, Y., Polo, L., Pérez, H., Gómez, R., Altamar, F., Álvarez, J., González, A. y Carbono, C. (2018). Fortalecimiento de hábitos de estudio a través de las tecnologías de la información y la comunicación. Cultura. Educación y Sociedad 9(3), 169-178. DOI: http://dx.doi.org/10.17981/cultedusoc.9.3.2018.20

\section{Resumen}

El propósito de la investigación correspondió a fortalecer el hábito a través del mejoramiento de la lectoescritura a partir de las Tecnologías de Información y Comunicación como herramienta estratégica. Desde los procesos investigativo derivados de la integración de la Investigación como Estrategia Pedagógica. Metodológicamente la investigación es de tipo cuantitativo- descriptivo. La unidad de análisis estuvo constituida por 60 estudiantes pertenecientes a los grados tercero y quinto, entre las edades de 7 a 12 años. Los resultados derivados dan cuenta de los procesos de mejoramiento y fortalecimiento de los hábitos de estudio en los estudiantes involucrados en el proceso investigativo a través del uso de las TIC. A través del proceso investigativo se puede concluir que como estrategia de enseñanza aprendizaje es positivo la implementación de las TIC a fin de mejorar los resultados obtenidos por los estudiantes en las pruebas locales y nacionales.

Palabras clave: Hábitos de estudio, tecnologías de la información y comunicación, lectura.

\section{Abstract}

The purpose of the research was to strengthen the habit through the improvement of literacy based on Information and Communication Technologies as a strategic tool. From the research processes derived from the integration of Research as a Pedagogical Strategy. Methodologically, the research is quantitative-descriptive. The analysis unit consisted of 60 students belonging to the third and fifth grades, between the ages of 7 to 12 years. The derived results show the processes of improvement and strengthening of study habits in students involved in the research process through the use of ICT. Through the research process it can be concluded that the implementation of ICTs is positive as a teaching-learning strategy in order to improve the results obtained by students in local and national tests

Keywords: Study habits, information and communication technologies, reading.

1 Este artículo ha sido derivado del Proyecto Fortalecimiento de la Cultura Ciudadana y Democrática en CT+I a través de la IEP apoyada en TIC en el Dpto. del Magdalena

2 Docentes de la Institución Educativa Departamental Rural de Media Luna, sedes Erm Varones De San José, colegio rural de media luna y Erm Niñas Del Socorro, del grupo de investigación "Inedermistas".

- The author; licensee Universidad de la Costa - CUC.

Cultura, Educación y Sociedad vol. 9 no. 3, pp. 169-178. Diciembre, 2018

Barranquilla. ISSN 2389-7724 Online 


\section{Introducción}

La población estudiantil de la institución educativa Departamental Rural de media luna, distribuida entre básica primaria y media se caracteriza por presentar un considerable bajo nivel en rendimiento académico, evidencia de ello son los resultados de las rubricas de prueba saber, las cuales describen anotaciones en rangos mínimos e insuficientes para más del $50 \%$ de los estudiantes en las pruebas descritas para los grados de $3^{\circ} 5^{\circ}$ y $9^{\circ}$ en las áreas evaluadas de lenguaje, ciencias naturales y matemáticas. Un denominador causal de la problemática se puede asociar a los inadecuados hábitos de estudio y las falencias en lectoescritura entre la población estudiantil, situación que genera dificultades para el oportuno proceso de formación personal al carecer en este sentido de habilidades de estudio entre los que destaca la comprensión lectora fundamental para un adecuado aprendizaje de todo estudiante entre las distintas áreas del conocimiento (Martín y Vera, 2012).

Por otro lado la práctica pedagógica docente tradicional de manera consciente o inconsciente se basa en un modelo conductista de enseñanza acogido a un método de educación estandarizado con poca aportación del estudiante desde su participación activa y de opinión; los anteriores elementos problematizadores podrían solucionarse mediante un proceso educativo en el cual se trabaje desde la práctica pedagógica espacios que generen una mayor motivación por aprender y desarrollar en consecuencia mejores hábitos de estudio apoyando en una buena comprensión lectora por parte de los estudiantes. Al respecto, autores como Mujica, García, Marín y Pérez (2006), resaltan la necesidad de una formación integral fundamentada en prácticas pedagógicas y profesionales que posibiliten un perfil de competencias centrado en lo cognitivo y actitudinal.
En este entendido, exponen Hernández y Jiménez (2015), que hoy día la sociedad "no reclama sólo la ética individual de las personas que componen las organizaciones, sino que ven un ente con responsabilidad propia económica, social y medioambiental a la que debe exigírsele comportamientos acordes a la misma" (p. 11), según su razón de ser.

Las organizaciones, señalan Mendoza, López y Salas (2016), "son entes que generan sus propios objetivos y diseñan la forma como alcanzarlos teniendo en cuenta, necesariamente, las influencias del entorno social" (p. 62). Las escuelas no escapan de ello y es de allí que, en las organizaciones, cualquiera que sea su ámbito, se hace preciso introducir una gestión estratégica como respuesta a las necesidades sociales. (Mendoza, López y Salas, 2016). Por tanto, expresa Cantillo (2013) la necesidad de incluir en las organizaciones programas de mejoramiento del ser, construyendo una cultura de estudios, resulta absolutamente necesaria.

Es así, como surge la necesidad de integrar los procesos participativos estudiantiles mediante la investigación en proyectos de aula que permitan ser abordados desde la práctica docente a partir de la Investigación como Estrategia Pedagógica (IEP), mejorando en este sentido las falencias descritas, respecto a la población de interés corresponde a estudiantes de la Institución rural de Media Luna, la cual se encuentra distribuida entre estudiantes de primaria y media con una población total de 842 estudiantes con quienes se trabajara una muestra de 287 estudiantes distribuidos entre $3^{\circ}$ (83 estudiantes), $6^{\circ} \mathrm{A}$ (26 estudiantes), $6^{\circ} \mathrm{B}$ (24 estudiantes), $6^{\circ} \mathrm{C}$ (20 estudiantes), $6^{\circ} \mathrm{D}$ (20 estudiantes), $5^{\circ}$ (85 estudiantes), y $8^{\circ}$ A (29 estudiantes) y $8^{\circ} \mathrm{B}$ (29 estudiantes) equivalentes a un $34 \%$ de la población estudiantil total. 


\section{Antecedentes}

Los procesos educativos son cada vez más cambiantes ante la transformación de las sociedades, por lo que cada día se requiere ver la escuela como un espacio de desarrollo personal y comunitario. El direccionamiento hacia una escuela de educación más contextualizada a la realidad social, depende del enfoque dirigido hacia la investigación como motor de aprehensión del interés estudiantil por aprender.

Según lo describe (González, 2008) la Pedagogía investigativa pretende direccionar los procesos educativos a una relación entre los seres humanos y cada espacio sociocultural, en el cual se pretende abarcar una interdisciplinariedad, en el que las actividades de formación permiten desarrollar actitudes y competencias para la construcción de los conceptos.

A nivel internacional se han publicado trabajos que describen propuestas de intervención que profundizan en la sistematización de la experiencia como estrategia generadora de conocimientos integrados a la formación crítico-reflexiva que permite que la persona aprenda mientras actúa en su rol profesional en el que como principal objetivo de este trabajo se ha evaluado y sistematizado un proceso de formación del docente como investigador, promovido a través de la práctica de la investigaciónacción (Aranguren, 2007; Medina y Zabalegui, 2010).

Para el anterior trabajo descrito por Aranguren en 2007 "La metodología aplicada es de tipo empírico, llevada a cabo mediante la estrategia de investigaciónacción, dada por las fases de exploración, planificación, experimentación, sistematización de experiencias y conocimientos. Se sistematizan tres ciclos y se hace énfasis en los aportes de cada actor en el desarrollo colectivo de su formación" (Aranguren, 2007). Dentro de la metodología que describe el trabajo se puede resaltar como ele- mento aportante para la presente investigación el "Plan Estratégico de Lectura y Escritura-Biblioteca", mediante el cual a través de lecturas dirigidas se trabajan los temas de interés para el proyecto escolar conduciendo en este sentido un mayor interés del estudiante por la temática trabajada.

Según Caldera, Escalante y Terán (2010) desde esta perspectiva la integración de la IEP al currículo permite ser una alternativa para encaminar la contribución a la solución de otros aspectos asociados a las características formativas de los estudiantes y de la manera en que estas experiencias contribuyen al acto pedagógico docente enfocado en el mejoramiento de los hábitos de estudio en los estudiantes desde el fortalecimiento de la lectoescritura. Conforme a lo planteado por Barnechea y Morgan (2010) el proyecto de investigación escolar anuncia sus propias metas u objetivos:

- Realizar consultas bibliográficas, relacionadas con la tradición, culturales y las costumbres de los pueblos.

- Construir una encuesta y una entrevista que permita conocer acerca de los juegos tradicionales, la economía familiar y el legado cultural de los habitantes de Media Luna. Construir y aplicar 3 encuestas (juegos tradicionales, economía familiar y legado cultural)

- Analizar los resultados de las encuestas, la entrevista y las descripciones de los lugares culturales. (Construir gráficos y conclusión de los resultados obtenidos

- Describir los lugares que hacen parte de la cultura y costumbres de Media Luna (parques, lugares de trabajo y lugares culturales). Realizar las visitas a los lugares que hacen parte de la cultura, costumbres y tradiciones, salida de campo. 
Los anteriores objetivos permiten a partir del interés común de la investigación generar en los estudiantes habilidades y mejoramiento en los hábitos de estudio, en especial desde el fortalecimiento personal en la búsqueda, interpretación y construcción de conocimientos, que derivan de la experiencia investigativa. Un perfil orientado al desarrollo de competencias investigativas en los estudiantes, con fundamento en los hábitos de estudio podrá ser fortalecido desde el acompañamiento de los diferentes actores, tal es el caso de la integración familiar, cuya dinámica y funcionalidad pasa a representar un agente catalizador para generar capacidades en sus integrantes. En este sentido, Dermarchi, Aguirre, Yela y Viveros (2015) conciben la dinámica familiar desde las múltiples interacciones y lazos que se establecen entre sus miembros; se infiere que las formas de relacionamiento dentro del núcleo familiar intervienen en la definición de las competencias personales.

\section{Metodología}

Desde el interés investigativo, el objeto de estudio de la investigación es de tipo cuantitativa, pues permite la identificación de un fenómeno, esto se da con el fin de verificar la influencia que tiene el proyecto en el resultado de los estudiantes en cuanto a sus hábitos de estudio frente a lectura.

Según el objetivo de la investigación es descriptiva, por lo tanto, expone al fenómeno a una precisión detallada de sus características, permitiendo la relación entre los elementos que hacen parte de este tener una información detallada.

\section{Participantes}

La unidad de análisis estuvo constituida por 60 estudiantes pertenecientes a los grados tercero y quinto, entre las edades de 7 a 12 años.

\section{Herramientas de recolección de} información

El proceso metodológico se inicia con un análisis diagnóstico del estado de las competencias a nivel de comprensión lectora en la población de estudio, en el cual se permita referenciar aspectos como la velocidad de lectura, calidad, comprensión lectora inferencial, literal y critica, pare ello se toma como referente los grados de $3^{\circ} \mathrm{y}$ $5^{\circ}$ de primaria y los resultados diagnósticos descritos por el Programa Todos a Aprender (PTA). (Castro y Páez, 2010).

Por otro lado, se continua con la revisión teórica de la ruta metodológica que describe la Investigación como Estrategia Pedagógico (IEP) apoyada en las Tecnologías de la Información y Comunicación (TIC), (momentos pedagógicos), mediante el cual se puedan dirigir proyectos de aula que permitan integrar al currículo, la IEP desde la creación de preguntas orientadoras derivadas del interés de los estudiantes, focalizadas en problemáticas contextualizadas a su realidad.

Como elemento de insumo se tomarán los temas de los proyecto de aula que se manejan en la institución educativa con los que se prevé trabajar lo relacionado a la lectura y escritura.

Respecto a las técnicas e instrumentos de recolección y organización de información, tener como eje central el uso de las TIC.

- Se plantea desde la colecta de información para valorar el estado de la comprensión lectura que deriva de los resultados de la evaluación realizada por el PTA en los cursos de $3^{\circ}$ y $5^{\circ}$ para las sedes de la Erm Niñas Del Socorro y Erm Varones De San José.

- Se plantea generar una pregunta problema entre la población de estudio, entorno a un tema de interés común; entre los cuales cabe resaltar el proyecto de aula que se maneja como experiencia investigativa desde la estrategia 1 del programa ciclón. 
- La pregunta de investigación seleccionada se trabajara desde las diferentes áreas del conocimiento.

- Los elementos de recolección de la información se realizan mediante la creación de relatorías individuales y grupales derivados de las lecturas dirigidas, mediante el uso de las TIC implementado experiencia en el transcurso de la investigación elaborada por los estudiantes, y colectada por los docentes investigadores y co-investigadores, para generar sus procesos reflexivos de la experiencia que le permita sistematizar el proceso.
Posteriormente desde la exploración y el recorrido de la trayectoria que describe la ruta metodológica de la IEP se pretende desde las diferentes áreas del conocimiento abordar lecturas dirigidas que permitan afianzar los procesos del aprendizaje por indagación, el aprendizaje colaborativo, situado y problematizador.

Para valorar el estado de la comprensión la lectura se deriva de los resultados de la evaluación realizada por el Programa Todos a Aprender (PTA) (MEN, 2012); en los cursos de $3^{\circ}$ y $5^{\circ}$ para las sedes de la Erm Niñas del Socorro y Erm Varones de San José, con forme los siguientes criterios.

TABLA 1

Criterio de valoración en la lectura.

\begin{tabular}{lll}
\hline VELOCIDAD & GRADO TERCERO & GRADO QUINTO \\
\hline Rápido & Por encima de 89 & Por encima de 124 \\
Óptimo & Entre 85 y 89 palabras & Entre 115 y 124 palabras \\
Lento & Entre 61 y 84 & Entre 100 y 114 \\
Muy lento & Por debajo de 60 & Por debajo de 100 \\
\hline
\end{tabular}

Fuente: MEN (CO), 2012.

TABLA 2

Criterios de clasificación para la definición de la calidad y comprensión de la lectura.

\section{CALIDAD} El (la) estudiante lee lentamente luchando con palabras que deben ser familiares, corta las
unidades de sentido largas (palabras y oraciones) y prima el silabeo.
B El (la) estudiante lee sin pausas ni entonación; lee las oraciones de un texto, palabra por palabra, sin respetar las unidades de sentido.
C En la lectura por unidades cortas el (la) estudiante ya une palabras formando oraciones con sentido, hace pausas, pero aún hay errores de pronunciación y entonación.
El (la) estudiante lee de forma continua, hace pausas y presenta inflexiones de voz adecuadas
D al contenido. Respeta las unidades de sentido y la puntuación. Se perciben menos errores de pronunciación. 


\begin{tabular}{cl}
\hline Cumple & $\begin{array}{l}\text { El (la) estudiante responde las dos preguntas de ubicación de información puntual de un } \\
\text { texto. }\end{array}$ \\
No & El (la) estudiante responde una o ninguna de las preguntas de ubicación de información \\
cumple & puntual de un texto. \\
\hline
\end{tabular}

\section{COMPRENSIÓN LECTORA (INFERENCIAL)}

\begin{tabular}{|c|c|}
\hline Cumple & $\begin{array}{l}\text { El (la) estudiante responde las dos preguntas donde debe relacionar la información para } \\
\text { hacer inferencias del texto. }\end{array}$ \\
\hline $\begin{array}{l}\text { No } \\
\text { cumple }\end{array}$ & $\begin{array}{l}\text { El (la) estudiante responde una o ninguna de las preguntas donde debe relacionar la } \\
\text { información para hacer inferencias del texto. }\end{array}$ \\
\hline
\end{tabular}

\section{COMPRENSIÓN LECTORA (CRÍTICA)}

\begin{tabular}{cl}
\hline Cumple & $\begin{array}{l}\text { El (la) estudiante responde las dos preguntas donde debe evaluar y reflexionar sobre el } \\
\text { propósito, el contenido o la forma del texto. }\end{array}$ \\
No & El (la) estudiante responde una o ninguna de las preguntas donde debe evaluar y reflexionar \\
cumple & sobre el propósito del texto, el contenido o la forma del texto. \\
\hline
\end{tabular}

Fuente: MEN (CO), 2012.

\section{Resultados}

Desde la experiencia de la práctica pedagógica en el que hacer docente, la aplicación de un mecanismo de integración curricular de la IEP, ha favorecido en gran medida el producto de la formación estudiantil. Es evidente que la implementación de contenidos curriculares novedosos vasados en proceso investigativos en el que los estudiantes a partir de experiencias investigativas, motivadas por preguntas que derivan del interés en común y de la necesidad colectiva de responder a un problema de contexto local, permite aterrizar el aprendizaje del estudiante sobre actividades que generen familiarización; resaltando además el papel de las nuevas tecnologías del aprendizaje, mediante el cual , toda la comunidad educativa en general se favorece.

Respecto a los resultados en el mejoramiento y o fortalecimiento de los hábitos de estudio en los estudiantes sobre la población de interés, se considera que existe un avance positivo dado que con la aplicación de las estrategias descritas anteriormente, se permitió mediar un mayor interés en el aprendizaje desde la resolución de la pregunta de investigación, motivando en este aspecto al estudiante a aprender de un tema a partir de las diferentes asignaturas en las que el proyecto pudo encontrar un contexto de aplicación, entre ellas las referentes a las ciencias sociales, naturales, filosofía, y leguaje.

Actualmente aun es un reto alcanzar un impacto masivo sobre la población de estudio respecto al desarrollo de habilidades y destrezas en los buenos hábitos de estudio, a partir del mejoramiento del componente lectoescritor del estudiante, en especial por que se requiere de una mayor atención por parte de los padres de familia a inmiscuirse en los procesos de aprendizaje de sus hijos que están en formación y que requieren de un acompañamiento continuo.

Conforme al primer instrumento derivado de las pruebas realizadas por el PTA para la población estudiantil de $3^{\circ}$ y $5^{\circ}$ de primaria se obtuvieron los siguientes resultados: 


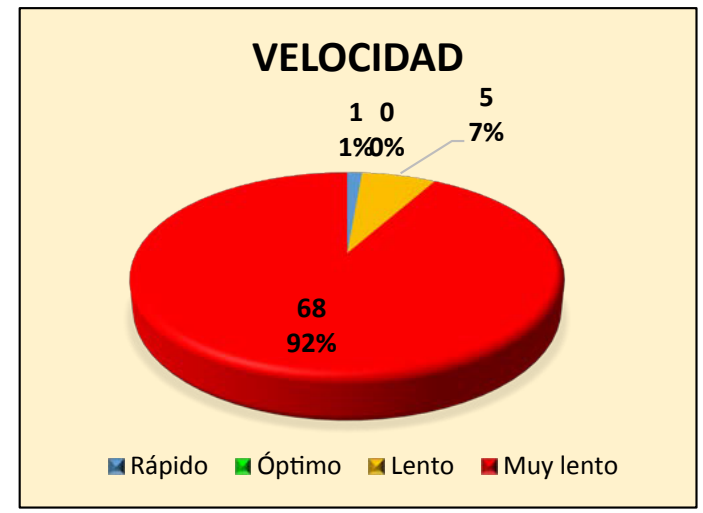

Figura 1. Relación porcentual de los resultados en el criterio de valoración en la velocidad de lectura de los estudiantes de $3^{\circ}$.

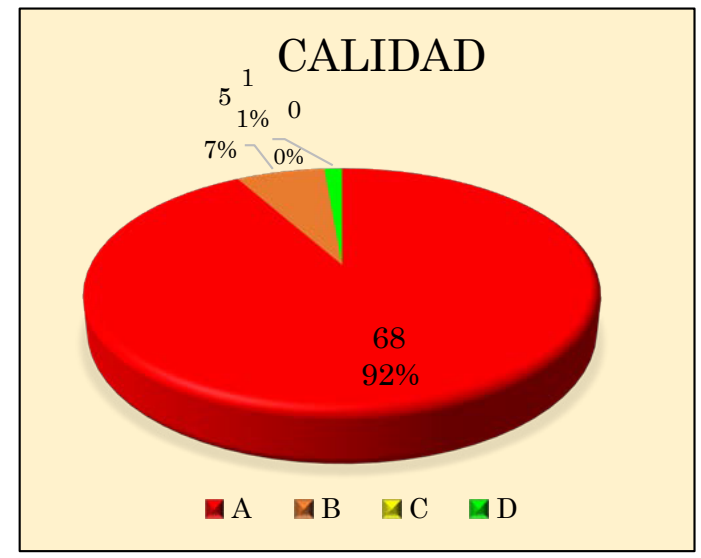

Figura 2. Relación de los resultados en el criterio de valoración en la calidad de la lectura de los estudiantes de los grados $3^{\circ}$.

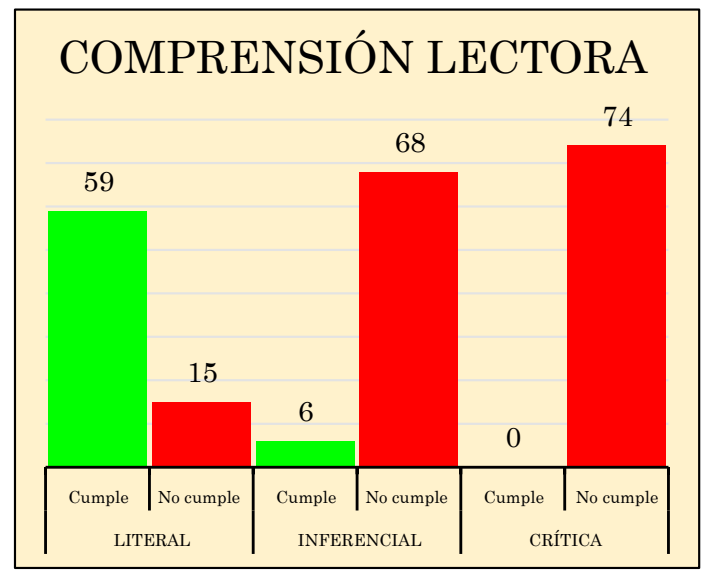

Figura 3. Relación de los resultados comparativos respecto a los criterios de comprensión lectora en estudiantes de $3^{\circ}$.

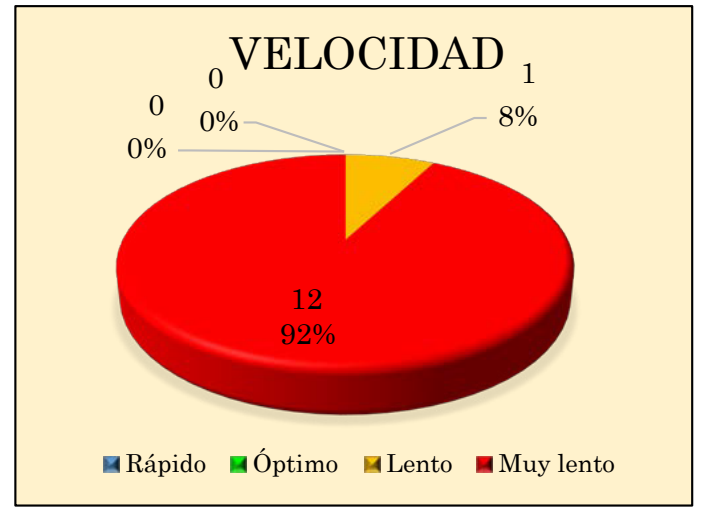

Figura 4. Relación porcentual de los resultados en el criterio de valoración en la velocidad de lectura de los estudiantes de $5^{\circ}$.

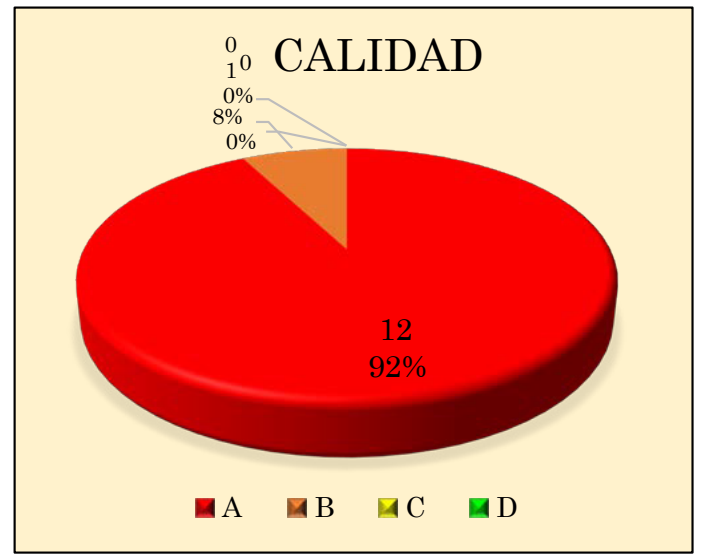

Figura 5. Relación de los resultados en el criterio de valoración en la calidad de la lectura de los estudiantes de los grados $5^{\circ}$.

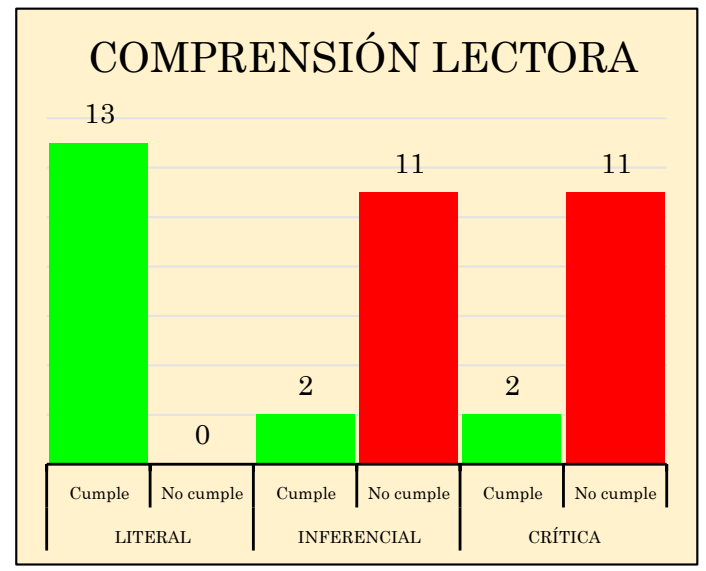

Figura 6. Relación de los resultados comparativos respecto a los criterios de comprensión lectora en estudiantes de $5^{\circ}$. 


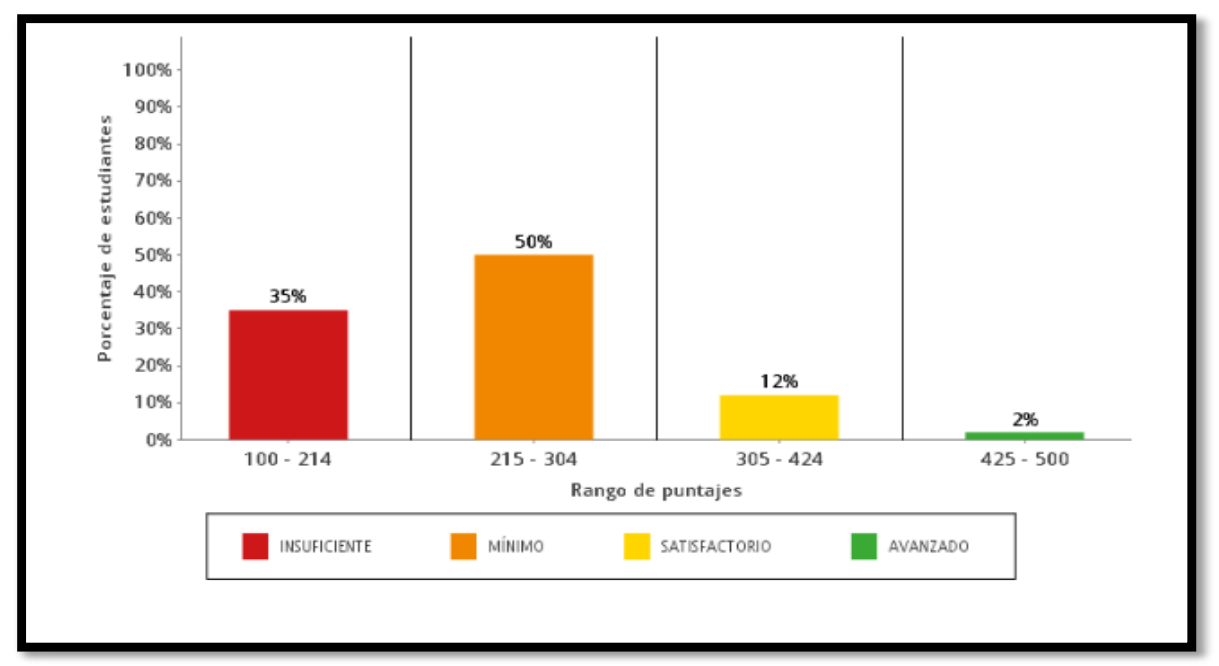

Figura 7. Porcentaje de estudiantes por niveles de desempeño en el establecimiento educativo. Lenguaje - grado noveno

Conforme a lo descrito en las anteriores figuras en la que se relacionan los resultados de la población de interés de $3^{\circ} 5^{\circ}$ y el grado $9^{\circ}$ respecto a las pruebas saber de este último correspondientes al año 2016, se permite inferir una denotación particularmente critica en lo referente al componente de la lectura y comprensión de texto, se puede interpretar que las dificultades en la calidad y la velocidad de lectura infieren en una disminución de la capacidad de interpretación inferencial y critica de lo que se está leyendo, esta situación permite identificar que el estudiante requiere de nuevos mecanismos que le permitan hacer una mayor práctica del ejercicio de la lectura así como de la escritura, porque si bien, el componente escrito no hizo parte del mecanismo preliminarmente evaluado en el PTA, sí se genera una correlación diciente de las dificultades que el estudiante puede tener para generar escritura .

Respecto a la integración curricular de la IEP desde el direccionamiento del proyecto escolar manejado desde la estrategia 1 del programa ciclón ver imagen 1 , se ha permitido contribuir desde la participa- ción activa estudiantil en la solución de un problema de interés común en el que el estudiante se hace responsable en un trabajo colaborativo, en el que a través de los procesos de indagación genera mejoras en las capacidades y o habilidades de la lectoescritura, permitiendo de este modo la exploración del estudiante hacia la creación de un progreso en los buenos hábitos de estudio, aplicables a la solución de problemas que este mismo pueda identificar desde la diferentes asignaturas que está cursando y desde este sentido les genera herramientas propias que le permiten un mejor desenvolvimiento en el entorno académico.

Además el desarrollo de actividades curriculares que permiten el uso de las herramientas informáticas en clase, favorece los mecanismos e instrumentos de consulta de la información para el desarrollo del proyecto de investigación ver imagen 2 . Convirtiéndose el uso de las TIC en otro elemento atrayente y facilitador en el desarrollo de habilidades de la lectoescritura y el consecuente fortalecimiento en los hábitos de estudio. 


\section{Discusión}

Los espacios de propagación de la experiencia investigativa corresponden a eventos de apropiación social del conocimiento, hasta la fecha respecto a la presente investigación aún no se realiza tal espacio, debido al corto tiempo con el que se dispone para el corte final de año lectivo, sin embargo, es importante resaltar la participación en estos espacios por parte del grupo de investigación constituido por estudiantes en distintos eventos, como lo son, las ferian institucionales y municipales, en los que se ha participado en conjunto con otras experiencias de investigación a nivel de otros temas, que corresponden a las áreas de medio ambiente y que hacen parte de los proyectos de investigación escolar de la institución en los que se describe una experiencia de integración curricular de la IEP.

\section{Referencias}

Aranguren, G. (2007). La investigaciónacción sistematizadora como estrategia de intervención y formación del docente en su rol de investigador. Revista de Pedagogía, 28 (82). Recuperado de h t t p : / / w w w . redal y c.org / pdf/659/65908202.pdf

Barnechea, M., y Morgan, M. (2010). La sistematización de experiencias: producción de conocimientos desde y para la práctica. Tend. Retos, 15, 97-107

Caldera, R., Escalante, D., y Terán, M. (2010). Práctica pedagógica de la lectura y formación docente. Revista de pedagogía, 31(88). Recuperado de http:// w w w. scielo.org.ve/scielo. php?script $=$ sci_arttext\&pid $=$ S0798-97922010000100002

Cantillo, J. (2013). Incidencia de la cultura organizacional en el desempeño. Económicas CUC, 34(1), 131-152.
Castro, A., y Páez, A. (2015). El mundo de la lectura: estrategias para la comprensión. [Tesis de maestría]. Recuperado de http://repositorio.uptc.edu.co/bitstream/001/1392/2/TGT-150.pdf

Demarchi, G., Aguirre, M., Yela, N., \& Viveros, E. (2015). Sobre la dinámica familiar. Revisión documental. Cultura Educación y Sociedad, 6(2). Recuperado de https://revistascientificas.cuc.edu.co/ culturaeducacionysociedad/article/ view/1049

Gonzales, J. (2008). Semilleros de investigación: una estrategia formativa. Revista Psychologia, Avances de la disciplina [en línea], 2 (2), 185-190. Recuperado de http://132.248.9.34/hevila/ PsychologiaAvancesdeladisciplina/2008/ vol2/no2/7.pdf

Hernández, H. y Jiménez, A. (2015). Responsabilidad social empresarial, zona urbana del distrito de Barranquilla: estudio etnográfico. Económicas CUC, $36(2), 9-18$.

ICFES. (2017). Publicación de resultados Saber $3^{\circ}, 5^{\circ}$ y $9^{\circ}$. Recuperado de http:// www2.icfesinteractivo.gov.co/ReportesSaber359/

Martin, M., y Vera, J. (2012). Dificultades en el aprendizaje de la lectoescritura en el primer ciclo de educación primaria. Intervención en el aula. [Trabajo de grado]. Recuperado de https://uvadoc.uva.es/ bitstream/10324/4828/1/TFG-L377.pdf

MEN (CO). (2012). Programa Todos a Aprender. República de Colombia. Consultado en https://www.mineducacion. gov.co/1621/articles-310659_archivo_ pdf_sustentos_junio27_2013.pdf

Medina, J. y Zabalegui, A. (2010). Entre la enseñanza y el aprendizaje. Un espacio de saberes para compartir. [Tesis doctoral]. Recuperado de: http://www.practicareflexiva.pro/w p-content/ uploads/2013/07/Tesis-Sevilla-4-DEFINITIVA-.pdf 
Mendoza, D., López, D. y Salas, E. (2016). Planificación estratégica de recursos humanos: efectiva forma de identificar necesidades de personal. Económicas CUC, 37(1), 61-79.

Mujica, M., Marín, F., Smith, H. y Lovera, M. (2006). Prácticas Profesionales Docentes y perfil Académico de Egreso: Estrategias para Contribuir al Desarrollo Productivo Regional. Multiciencias, 6(2), 162-167

Patiño, A. (2015). Tendencias tecnológicas que influyen en el aumento de la productividad empresarial. INGE CUC, 11(2), 84-96. https://doi.org/10.17981/ ingecuc.11.2.2015.09
Sepulveda, L. (2014). La incorporación de la tecnología en la enseñanza de la química. [Tesis de pregrado]. Recuperado de http://bibliotecadigital.univalle.edu.co/ bitstream/10893/7189/1/3467-0430907. pdf

Spina, M., Rohvein, C., Urrutia, S., Roark, G., Paravié, D. y Corres, G. (2016). Aplicación del modelo SCOR en pymes metalmecánicas de Olavarría. INGE CUC, 12(2), 50-57. https://doi.org/10.17981/ingecuc.12.2.2016.05 